\title{
De Novo Formation and Rupture of an Intracranial Aneurysm 10 Months After Normal Findings on Conventional Magnetic Resonance Angiography in a Patient With No History of Intracranial Lesions
} -Case Report-

\author{
Toshiyuki OKAZAKI, Toru NiSHI, Shigeo YAMASHIRO, \\ Kazunari KOGA, Shinji NAGAHIRO*, and Shodo FUJIOKA \\ Department of Neurosurgery, Stroke Center, Saiseikai Kumamoto Hospital, Kumamoto; \\ *Department of Neurosurgery, School of Medicine, The University of Tokushima, Tokushima
}

\begin{abstract}
A 40-year-old male smoker presented with a ruptured saccular aneurysm that formed de novo 10 months after normal magnetic resonance (MR) angiography findings. Computed tomography of the head at the second admission showed subarachnoid hemorrhage in the interhemispheric fissure and anterior basal cistern. MR and conventional cerebral angiography revealed an aneurysm arising from the anterior communicating artery (Acom A). MR angiography performed 10 months earlier, when he experienced transient loss of consciousness, revealed no anomalies at the AcomA. Intraoperatively, the de novo aneurysm was found to be a typical saccular aneurysm with a fragile wall.
\end{abstract}

Key words: de novo aneurysm, magnetic resonance angiography, subarachnoid hemorrhage, early formation, anterior communicating artery

\section{Introduction}

Aneurysms confirmed to be the result of de novo formation are described as de novo aneurysms, $2,8,11,13,16,18,19,23-25)$ and are usually detected by conventional cerebral an- giography at follow up or examination for new subarachnoid hemorrhage (SAH) after intracranial surgery. Age, female sex, smoking, and hypertension are risk factors for the formation of de novo aneurysms. ${ }^{6,7)}$ Local hemodynamic changes due to earlier surgical interven-

Received May 28, 2009; Accepted August 20, 2009

Author's present address: T. Okazaki, M.D., Department of Neurosurgery, School of Medicine, The University of Tokushima, Tokushima, Japan. 
tion, ${ }^{22)}$ local fragility of the arterial wall, arterial wall damage attributable to the application of temporary clips or the edge of permanent clips, and earlier gamma knife surgery are all associated with de novo aneurysms. ${ }^{14)}$ Most cases of de novo aneurysms develop within a few years of intervention, and development within 1-2 months is rare. ${ }^{16,18,24)}$

We treated a patient with de novo cerebral aneurysm which ruptured within 10 months of the acquisition of normal magnetic resonance (MR) angiography findings.

\section{Case Report}

A 40-year-old male smoker was first seen at our hospital with transient loss of consciousness after alcohol consumption. He did not complain of headache. Head computed tomography (CT), MR imaging, MR angiography, and Holter electrocardiography yielded no abnormal findings with respect to the cranial structures including the anterior communicating artery (AComA) (Fig. 1A). Our diagnosis was post-alcohol syncope. He was symptom-free for the next 10 months but was re-admitted with severe headache and nausea about 4.5 hours after experiencing onset of headache while voiding his bowels. No neurological deficits were identified, but head CT revealed SAH in
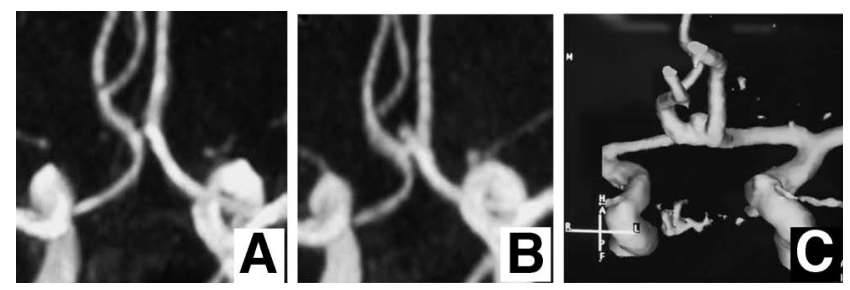

Fig. 1 A: Magnetic resonance (MR) angiogram showing no anomalies in structures including the anterior communicating $\operatorname{artery}(\operatorname{ACom} A)$ at first admission. B: Two-dimensional MR angiogram showing a de novo aneurysm arising from the AComA after the diagnosis of subarachnoid hemorrhage (SAH). C: Three-dimensional MR angiogram after the diagnosis of SAH.

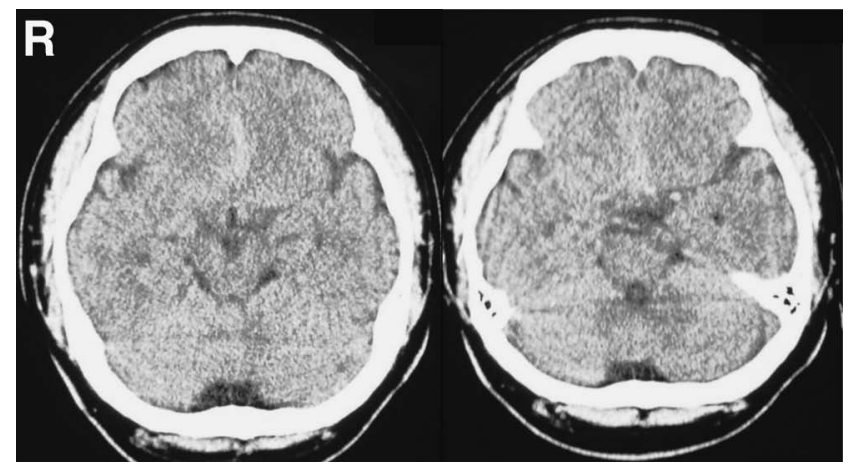

Fig. 2 Computed tomography scans obtained at the second admission demonstrating subarachnoid hemorrhage localized to the interhemispheric fissure and anterior basal cistern. the interhemispheric fissure and anterior basal cistern (Fig. 2). Conventional cerebral angiography (Fig. 3), and two-dimensional (2D) and three-dimensional (3D) MR angiography (Fig. 1B, C) confirmed formation of a de novo aneurysm arising from the previously normal AComA, acquired at the same imaging facility and with the parameters applied on first admission. The left anterior cerebral artery was slightly dominant and the diagnosis was SAH caused by aneurysm rupture.

He had no history of meningitis, head injury, other intracranial diseases, or family history of SAH or unruptured cerebral aneurysm. The only known risk factor was smoking history. The aneurysm was clipped with a Sugita No. 89 mini-clip through the trans-sylvian approach. Operative findings demonstrated a typical saccular aneurysm with a fragile neck (Fig. 4). MR imaging showed neither dissecting nor thrombosed aneurysm. Cerebral angiography confirmed complete aneurysm clipping and his postoperative course was uneventful. Based on his clinical course, we conclude that a de novo aneurysm spontaneously developed and ruptured within 10 months of the first admission.

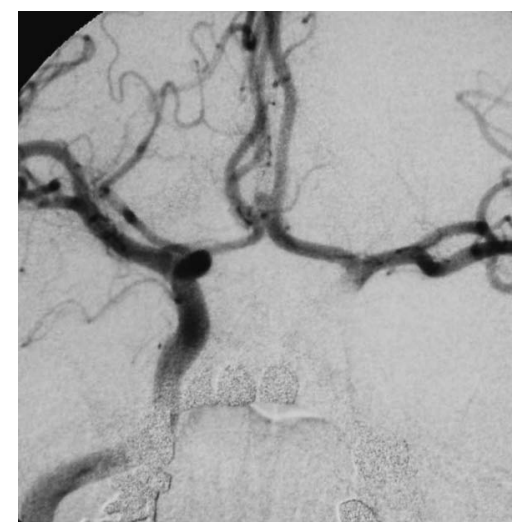

Fig. 3 Right common carotid angiogram with compression of the left common carotid artery.

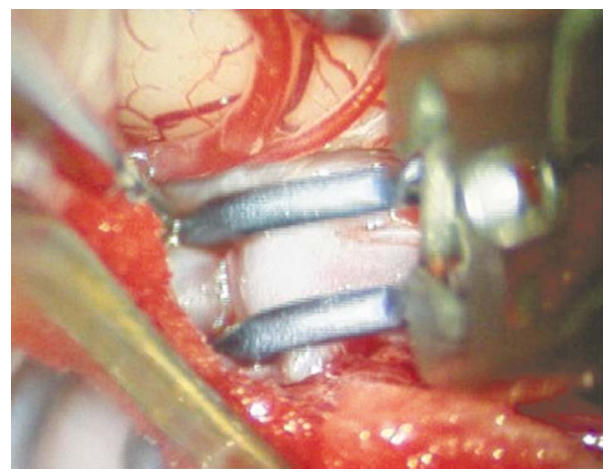

Fig. 4 Intraoperative photograph confirming that the de novo aneurysm was a saccular aneurysm with a clippable neck arising from the anterior communicating artery. 


\section{Discussion}

The probability of de novo aneurysm formation is $0.84 \% / y e a r,{ }^{6)}$ and the major risk factors in the development of de novo aneurysms are female sex and smoking. In general, the risk factors for aneurysm rupture are smoking, hypertension, and heavy alcohol consumption. ${ }^{15,20,21)}$ In our patient, the only known risk factor for aneurysm formation and $\mathrm{SAH}$ was a history of smoking.

De novo aneurysms commonly occur in the third and fourth decades of life with an interval of $3-20$ years. ${ }^{21)}$ Rapid development and rupture of de novo aneurysms within 1 year is known, ${ }^{1,11,13,16,18,24)}$ with the shortest reported interval of 10 days. ${ }^{1)}$ Proposed hypotheses for the development and rupture of de novo aneurysms include earlier intracranial aneurysm surgery leading to changes in the balance and periodicity of pulsatile collateral flow that allow the development of aneurysms at fairly predictable sites, ${ }^{3,17)}$ and the persistence of the same inherent defect(s) that allowed the development of the earlier aneurysm. ${ }^{12)}$ False-negative results in the detection of aneurysms must also be considered. All previously reported patients with rapidly developing de novo aneurysms had a past history of SAH caused by intracranial aneurysm rupture..$^{1,11,13,16,18,24)}$ In contrast, the present patient had no past history of SAH.

Unequivocal identification of a de novo aneurysm must exclude the presence of a residual aneurysm at the site. The diagnosis of previous cases was based on conventional cerebral angiography. In our case, aneurysm rupture was detected on 2D and 3D MR angiography and conventional angiography obtained after $\mathrm{SAH}$ onset at the second admission. Only 2D MR angiography was performed at the first admission. Artifacts such as turbulent and/or slow blood flow may return false-negative results on 2D MR angiography performed for the detection of internal carotid artery and AComA aneurysms. ${ }^{18,24)}$ However, since we obtained both sets of 2D MR angiograms at the same facility, using the same instrument and identical scanning parameters ( 1.5 tesla, echo time $3.4 \mathrm{msec}$, repetition time $38 \mathrm{msec}$ ), we conclude that the ruptured aneurysm did not exist at the time of the first MR angiography study.

Rats subjected to hemodynamic stress and hypertension manifested aneurysmal changes at the anterior and olfactory artery bifurcation within 3 months. ${ }^{4)}$ The rate of these changes was higher in estrogen-deficient female rats with hypertension and hemodynamic stress, ${ }^{5}$ and endothelial dysfunction and strong oxidative stress were observed in the aneurysm wall. Hormone replacement with $17 \beta$ estradiol or treatment with angiotensin type 1 receptor blocker (ARB) reduce the incidence of human aneurysm formation. ${ }^{24)}$ Estrogen has anti-oxidative, anti-inflammatory, and vasoprotective effects in addition to the antihypertensive actions. ARB also produces similar effects. Therefore, we propose that oxidative stress, inflammation, and hypertension contribute to acute aneurysm formation. The anti-protease activity of alpha 1-anti-trypsin (alpha 1-AT) is reduced in patients with SAH caused by intracranial aneurysm rupture. Alpha 1-AT is highly sensitive to oxidant agents, particularly in patients who con- tinue to smoke. ${ }^{10)}$ Moreover, smoking results in acute increase in blood pressure lasting for approximately 3 hours. ${ }^{9)}$ Bowel movement may also produce a transient increase in blood pressure.

Based on these considerations, we postulate that the vascular wall in our patient was exposed to chronic oxidative stress and transient hypertension due to his continued smoking, and that weakening of his anti-oxidative defense systems and the dominance of the anterior cerebral artery may have contributed to the formation and rupture of the de novo aneurysm.

The present case suggests that even in the absence of risk factors such as a history of intracranial surgery or a family history of SAH, a de novo aneurysm can be acquired and rupture to cause SAH in as little as 10 months. This has important implications with respect to the brain check-up systems used in Japan that employ MR angiography as the most common diagnostic modality for cerebral aneurysm screening. Common risk factors must be considered even in patients with normal MR angiography screening results, and shortening of the interval between screening studies for cerebral aneurysms may be advisable in symptomatic individuals.

\section{References}

1) Abe T, Saito N, Kunishio K: [De novo aneurysm after ten days from the onset of SAH]. No Shinkei Geka 36: 1109-1113, 2008 (Jpn, with Eng abstract)

2) Brock S, Giombini S, Ciceri E: Development and rupture of a de novo basilar artery aneurysm after surgical removal of a cerebellar arteriovenous malformation. Acta Neurochir (Wien) 145: 1117-1120, 2003

3) Crompton MR: The pathogenesis of cerebral aneurysms. Brain 89: 797-814, 1966

4) Jamous MA, Nagahiro S, Kitazato KT, Satoh K, Satomi J: Vascular corrosion casts mirroring early morphological changes that lead to the formation of saccular cerebral aneurysm: An experimental study in rats. J Neurosurg 102: 532-535, 2005

5) Jamous MA, Nagahiro S, Kitazato KT, Tamura T, Kuwayama K, Satoh K: Role of estrogen deficiency in the formation and progression of cerebral aneurysms. Part II: Experimental study of the effects of hormone replacement therapy in rats. J Neurosurg 103: 1052-1057, 2005

6) Juvela S, Poussa K, Porras M: Factors affecting formation and growth of intracranial aneurysms: A long-term followup study. Stroke 32: 485-491, 2001

7) Juvela S, Porras M, Poussa K: Natural history of unruptured intracranial aneurysms: Probability of and risk factors for aneurysm rupture. J Neurosurg 108: 1052-1060, 2008

8) Kim do H, Jung JY, Lee JW, Huh SK, Lee KC: A clinical analysis of twelve cases of ruptured cerebral de novo aneurysms. Yonsei Med J 48: 30-34, 2007

9) Longstreth WT Jr, Nelson LM, Koepsell TD, van Belle G: Cigarette smoking, alcohol use, and subarachnoid hemorrhage. Stroke 23: 1242-1249, 1994

10) Marzatico F, Gaetani P, Tartara F, Bertorelli L, Feletti F, Adinolfi D, Tancioni F, Rodrigues y Baena R: Antioxidant status and alpha 1-antiproteinase activity in subarachnoid hemorrhage patients. Life Sci 63: 821-826, 1998

11) Matheus MG, Castillo M: Development of de novo intracranial aneurysm in three months: Case report and litera- 
ture review. AJNR Am J Neuroradiol 24: 709-710, 2003

12) Miller CA, Hill SA, Hunt WE: "De novo" aneurysms. A clinical review. Surg Neurol 24: 173-180, 1985

13) Obray R, Clatterbuck R, Olvi A, Tamargo R, Murphy KJ, Gailloud P: De novo aneurysm formation 6 and 22 months after initial presentation in two patients. AJNR Am J Neuroradiol 24: 1811-1813, 2003

14) Park KY, Ahn JY, Lee JW, Chang JH, Huh SK: De novo intracranial aneurysm formation after gamma knife radiosurgery for vestibular schwannoma. J Neurosurg 110: 540-542, 2009

15) Sankai T, Iso $H$, Shimamoto $T$, Kitamura $A$, Naito $Y$, Sato $S$, Okamura T, Imano H, Iida M, Komachi Y: Prospective study on alcohol intake and risk of subarachnoid hemorrhage among Japanese men and women. Alcohol Clin Exp Res 24: 386-389, 2000

16) Schebesch KM, Doenitz C, Zoephel R, Finkenzeller T, Brawanski AT: Recurrent subarachnoid hemorrhage caused by a de novo basilar tip aneurysm developing within 8 weeks after clipping of a ruptured anterior communicating artery aneurysm: Case report. Neurosurgery 62: E259-260, 2008

17) Sheffield EA, Weller RO: Age changes at cerebral artery bifurcations and the pathogenesis of berry aneurysms. J Neurol Sci 46: 341-352, 1980

18) Sim JH, Kim SC, Kim MS: Early development and rupture of de novo aneurysm-Case report. Neurol Med Chir (Tokyo) 42: 334-337, 2002

19) Stiefel MF, Al-Okaili R, Weigele JB, Hurst RW: De novo aneurysm formation and regression after brain ar- teriovenous malformation embolization: Case report. Surg Neurol 67: 99-101, 2007

20) Tuomilehto J, Sarti C, Narva EV, Salmi K, Sivenius J, Kaarsalo E, Salomaa V, Torppa J: The FINMONICA Stroke Register. Community-based stroke registration and analysis of stroke incidence in Finland, 1983-1985. Am J Epidemiol 135: 1259-1270, 1992

21) van Gijn J, Rinkel GJ: Subarachnoid haemorrhage: Diagnosis, causes and management. Brain 124: 249-278, 2001

22) Wang YY, Rosenfeld JV, Lyon SM, O'Brien BJ: Rapid development of a de novo intracranial aneurysm following carotid occlusion. J Clin Neurosci 15: 324-330, 2008

23) Wermer MJ, Rinkel GJ, Greebe P, Albrecht KW, Dirven CM, Tulleken CA: Late recurrence of subarachnoid hemorrhage after treatment for ruptured aneurysms: Patient characteristics and outcomes. Neurosurgery 56: 197-204, 2005

24) Yasuhara T, Tamiya T, Sugiu K, Inoue S, Ohmoto T: De novo formation and rupture of an aneurysm. Case report. J Neurosurg 97: 697-700, 2002

25) Yoneoka Y, Takeda N, Akira I, Ibuchi Y, Kumagai T, Sugai $\mathrm{T}$, Takeda KI, Ueda K: Ruptured de novo intracranial aneurysms. Acta Neurochir (Wien) 146: 979-981, 2004

Address reprint requests to: Toshiyuki Okazaki, M.D., Department of Neurosurgery, School of Medicine, The University of Tokushima, 3-18-15 Kuramoto-cho, Tokushima, Tokushima 770-8503, Japan.

e-mail: tokazaki1978@sirius.ocn.ne.jp 